\title{
EXPLORANDO NECESIDADES OCUPACIONALES: UN ESTUDIO DE CASO
}

\section{Exploring Occupational Needs: A study of case}

\author{
Antonia Echeverría Ruiz-Tagle ${ }^{\mathrm{i}}$. \\ Pilar Ortega Lerdo de Tejada ${ }^{\text {ii }}$ \\ Viviana Riquelme Echeverría. iii
}

\section{RESUMEN}

Este trabajo, da conocer la definición operacional de Necesidad Ocupacional aplicada en la investigación de Seminario de Título: Necesidades Ocupacionales de una madre cuidadora de una NANEAS (Niño o Adolescente con Necesidades Especiales de Atención en Salud) durante la hospitalización. La muestra utilizada correspondió a la madre de una NANEAS hospitalizada en el Hospital Clínico de la Universidad de Chile, durante el año 2007-2008. Los datos obtenidos del estudio de caso fueron revaluados, por ende difieren del estudio anterior. Tras el análisis pudimos constatar que las áreas del desempeño mayormente vulneradas en esta madre, durante los periodos de hospitalización fueron: las AVD instrumentales, Trabajo y Tiempo Libre.

La relevancia de este artículo se basa en la entrega de conocimiento y técnicas (Cuestionario de Necesidad Ocupacional) que contribuyen al desarrollo de la Ciencia de la Ocupación, y su práctica, la Terapia Ocupacional.

El proceso de identificación de las Necesidades Ocupacionales (de ahora en adelante NN.OO), es una herramienta de diagnóstico, planificación, ejecución, evaluación y sistematización en salud que permite crear líneas de acción, convirtiéndose en un medio terapéutico de inclusión.

Palabras claves: Necesidad Ocupacional, brecha ocupacional, sentimiento de satisfacción de vida, ocupaciones significativas, Cuestionario de Necesidad(es) Ocupacional(es).

\section{ABSTRACT}

The purpose of this article is to explain the meaning of Occupational Need after it's appliance during the seminaries' investigation: “Occupational Needs of a Caregiver Mother during a Child with Special Health Care Need's hospitalization.”

\footnotetext{
' Lic. en Ciencia de la Ocupación, Terapeuta Ocupacional, Encargada Programa de Inclusión Laboral FONADIS, antoniaecheverriart@gmail.com.

ii Lic. en Ciencia de la Ocupación, Terapeuta Ocupacional. Terapeuta ocupacional Equipo de Psiquiatría Comunitaria Hospital Barros Luco, pilar.ortega.ldt@gmail.com.

iii Terapeuta Ocupacional. Lic. en Ciencia de la Ocupación, Terapeuta Ocupacional, Profesora Asistente Escuela de Terapia Ocupacional. Universidad de Chile. vriquelme@med.uchile.cl. Fono 9786183
} 
For the investigation, there was observed the Child with Special Health Care Need's mother hospitalized in the Universidad de Chile's University along the period 2007 - 2008.

The data of the case study has been reevaluated, that is why it varies from the previous study. After the analysis, it has been detected that the performance areas that were mostly injured during the hospitalization period were: the instrumental activities of daily living, work and leisure.

The relevancy of this study is based on the acknowledgement and techniques (Occupational Needs Questionnaire) contribution to the development of the Occupational Science and it's practice: the Occupational Therapy.

The identification process of the Occupational Needs, is a tool for diagnosis, planning, execution, evaluation and systematization for health that allows to create action lines, turning itself into an inclusive therapeutic mean.

Key-words: Occupational Needs, Occupational gap, life satisfaction, meaning occupations, Occupational Needs Questionnaire

\section{INTRODUCCIÓN}

El ser humano, a lo largo de su historia ocupacional va construyendo su identidad y definiendo su estilo de vida a través de su comportamiento ocupacional. A medida que crece, se desarrolla y madura, adquiere experiencias de vida que le permiten proyectarse a futuro en relación a lo que le gustaría ser, hacer y en donde le gustaría estar.

La necesidad ocupacional (de ahora en adelante N.O.) surge de la reflexión personal del individuo, el cual ante un proceso de introspección denota una alteración en el sentimiento de satisfacción de vida producto de una brecha ocupacional (brecha que ocurre entre lo que un individuo quiere y necesita hacer, y lo que él/ella realmente hace). Mientras la persona no sea consciente de cómo ésta brecha está generando modificaciones en su participación y desempeño, no adoptará una actitud activa que le permita invertir energías en pos de un cambio. En otras palabras, al percibir la N.O. y el impacto que ésta produce en su desempeño, su salud, calidad de vida y en su entorno, la persona se motiva a realizar un cambio a nivel del ser, hacer y/o estar.

El concepto como tal, surge durante el estudio de una madre cuidadora de una NANEAS ${ }^{1}$, (Niños/as y/o Adolescentes con Necesidades Especiales de Atención en Salud) durante su periodo de hospitalización. Dicha investigación, realizada durante el 2007 - 2008, permitió crear la definición operacional de Necesidad Ocupacional y el Cuestionario de Necesidades Ocupacionales.

Tras el análisis pudimos constatar que las áreas del desempeño mayormente vulneradas durante los periodos de hospitalización fueron: las AVD instrumentales, Trabajo y Tiempo Libre. Las AVD básicas son cubiertas casi en su totalidad. El desempeño en el área de Participación Social es modificada y se perciben nuevas NN.OO. en Educación. 
El siguiente trabajo pretende mostrar los resultados obtenidos tras un proceso de reevaluación de los datos recopilados en el Seminario de Titulo, dando a conocer el concepto e instrumentos aplicados, junto a su fundamentación teórica.

\section{MARCO CONCEPTUAL}

El concepto de Necesidad Ocupacional está cimentado en las bases en el Marco de Trabajo: dominio y procesos para la T.O., en el Modelo Canadiense del Desempeño Ocupacional ${ }^{2}$ y el Modelo de Ocupación Humana ${ }^{3}$ y la Teoría de Comportamiento Ocupacional de Mary Reilly.

Para comprender los fundamentos que engloban al concepto en sí y a su definición conceptual, revisaremos en detalle su evolución.

La práctica centrada en el cliente del Modelo Canadiense de Desempeño Ocupacional, modifica la actitud pasiva de la persona en el proceso de rehabilitación, haciendo un cambio de mando. El Modelo Canadiense, deja de ver a la persona como paciente (rol pasivo) y lo posiciona como un cliente o sujeto de derecho (rol activo). Esta transformación en la concepción, se traduce en un cambio en nivel de interacción, es decir, de ser un receptor de información y someterse a las indicaciones de los expertos, comienza a ser un actor en el proceso de rehabilitación y cambio, tomando sus decisiones en base a las necesidades que éste percibe como significativas dentro de su historia ocupacional. El usuario tiene la responsabilidad y poder para identificar sus necesidades y generar cambios. El terapeuta ocupacional (T.O) y profesionales a cargo de este proceso, son guías y facilitadores que le enseñarán a la persona estrategias para lograr alcanzar la meta deseada, y así satisfacer la necesidad percibida. Lo anterior es lo que el modelo denomina como "empoderamiento", agente movilizador al cambio en cuanto la persona es un ser activo que puede modificar su historia ocupacional al involucrarse en ocupaciones significativas que estén acorde a su situación personal y/o contextual. Para ello el Modelo la Medición Canadiense de Desempeño Ocupacional (COPM), el cual permite a la persona evaluar las ocupaciones de la vida diaria que conforman su rutina, valorándolas en base a tres criterios: el nivel de importancia en relación a las actividades y ocupaciones que conforman su rutina, nivel de satisfacción que le produce dicha ocupación y el nivel de desempeño.

En el Marco de Trabajo para la Práctica de la T.O. ${ }^{4}$, establece una guía de referencia para el quehacer profesional. Al ser un documento creado en base a la Clasificación Internacional del Funcionamiento, de La Discapacidad y de La Salud (CIF), otorga una mirada actualizada e integral de los lineamientos profesionales y lo que implica para la Terapia Ocupacional el desempeño ocupacional del ser humano, el proceso de evaluación e intervención ocupacional.

En la evaluación el foco de atención está centrado en crear el perfil ocupacional de la persona y en base a éste analizar el desempeño. Si bien este proceso está dividido en dos etapas (perfil ocupacional y análisis del desempeño ocupacional), éstas suelen interponerse; es decir, mientras la persona expresa y/o manifiesta su quehacer cotidiano, el T.O. construye la historia ocupacional en conjunto con la persona. Por lo tanto, la persona en conjunto con el T.O. realizan un análisis de su desempeño ocupacional a lo largo del ciclo vital. 
Lo esencial del proceso de evaluación, radica en la percepción de necesidades, problemas y preocupaciones que identifica la persona acerca de su desempeño ocupacional y actividades que conforman su rutina diaria, al hacer un análisis de su historia ocupacional y del desempeño que ha tenido en ésta. Lo anterior le permite establecer sus prioridades, gracias al proceso de introspección y reflexión. A su vez, al T.O. le permite identificar los factores protectores y los factores de riesgo (reales o potenciales) que apoyan o dificultan dicho desempeño, entregando los lineamientos para el diseño del plan de tratamiento.

El Modelo de Ocupación Humana, considera al ser humano como un sistema abierto, que es capaz de recibir o percibir un estímulo (intake/input), procesarlo (throughout) y generar una respuesta que se traduce en un determinado comportamiento (output). Pero desde que se procesa el estímulo hasta que éste se lleva a cabo a través del comportamiento, el ser humano tiene la posibilidad de modificarlo al recibir retroalimentación del medio ambiente que lo rodea (feedback). Gracias a este ciclo, la persona logra experimentar aprendizajes. $\mathrm{Al}$ involucrarse en ocupaciones el ser humano desarrolla su comportamiento ocupacional en base a las experiencias vividas y a las oportunidades de exploración, manipulación y dominio que le brinda el contexto en donde se desenvuelve. A medida que va adquiriendo un sentimiento de logro y dominio, éste le permite visualizarse como un ser competente capaz de involucrarse en actividades y ocupaciones nuevas y que son de su interés., 6

El ser humano, al ser un ser ocupacional que interactúa constantemente con el ambiente por medio de una ocupación, vive en un continuo proceso de adaptación para satisfacer sus necesidades de acuerdo a las características del entorno y a las exigencias socio-culturales de éste. ${ }^{7}$ El comportamiento ocupacional a lo largo del ciclo vital determina en cada una de las etapas ciertas tareas evolutivas que el ser humano requiere satisfacer para alcanzar o pasar a la siguiente etapa.

Para lograr adaptarse a las modificaciones ambientales que permanentemente sufre el entorno, modifica su participación en las ocupaciones de la vida diaria, eliminando de su rutina algunas actividades o modificando el modo de desempeño. En otras palabras, para que un ser humano logre desenvolverse y adaptarse a los diferentes contextos, ha de tener flexibilidad y efectividad para dar respuesta y satisfacer las demandas ambientales.

Kielhöfner define causalidad personal como "la iniciación (por parte del individuo) del comportamiento destinado a producir un cambio en el ambiente" ${ }^{7}$, entendiéndola como un paso posterior a la identificación y percepción de una brecha que requiere ser satisfecha. Se deduce entonces, que la causalidad personal depende de la participación del ser humano en las ocupacionales de la vida diaria, puesto que en base a éstas será la modificación en el comportamiento ocupacional, que en última instancia determinará su desarrollo como ser ocupacional.

Aun cuando el ser humano como tal, tiende a generar cambios en su comportamiento a lo largo de su historia de vida, la causalidad es gatillada por la combinación simultánea de una serie de estímulos en conjunto con la alteración con el sentimiento de satisfacción de vida, que interactúan en un momento y espacio determinado. Ante lo cual, si bien el locus de causalidad descansa dentro del individuo, no es el único elemento en juego al momento de generar un cambio en el desempeño de la rutina.

\section{Definición Operacional de Necesidad Ocupacional}

En el marco de esta investigación se realizó un proceso de construcción y análisis conceptual que concluyó en la definición de N.O. descrita a continuación. 
Necesidad Ocupacional ${ }^{8}$ "es la percepción de una brecha ocupacional; que altera el sentimiento de satisfacción de vida, modificando la participación de la persona en el desempeño de ocupaciones significativas".

\section{Conceptos claves involucrados en esta definición:}

\section{Percepción}

Ricardo Caponni, en su libro Psicopatología y Semiología Psiquiátrica, define percepción como "el acto de toma de conocimientos de datos sensoriales del mundo que nos rodea. Ésta constituye un mecanismo a través del cual el hombre adquiere el conocimiento del mundo exterior, o de su propio mundo interior.

En cuanto a lo percibido adquiere significación para nosotros, adquiere un contenido significativo y pasa a la categoría de vivencia perceptiva. Los determinantes de tal contenido significativo de la percepción, son múltiples. Uno de los fundamentales es la afectividad. El estado emocional, los sentimientos y el estado de ánimo organizan nuestra percepción, en el fondo, vemos lo que esperamos ver. En el mismo sentido la experiencia previa en el trayecto vital, nuestra biografía, condiciona la percepción. Estamos más entrenados para percibir algunas cosas y otras no. De lo percibido, algunos elementos pasan a primer plano y estructuran la figura perceptiva." ${ }^{9}$

Dentro de las principales características de la percepción según Caponni, es su carácter subjetivo, íntimamente relacionado con la afectividad, estado emocional e historia ocupacional de la persona. De esta manera, se puede explicar el por qué las NN.OO. difieren de una persona a otra dentro de un mismo contexto socio-cultural.

En la medida que percibimos y otorgamos significado a nuestro desempeño en las distintas ocupaciones de la vida diaria, nos percatamos de aquellas ocupaciones que para nosotros son significativas y por tanto adquieren importancia y nos proporcionan satisfacción en la medida que las desempeñamos adecuadamente.

\section{Brecha ocupacional}

En la Tesis "Occupational gaps after acquired brain injury: An exploration of participation in everyday occupations and the relation to life satisfaction", de Gunilla Eriksson. Se establece como brecha ocupacional a "brecha que ocurre entre lo que un individuo quiere y necesita hacer, y lo que él/ella realmente hace." ${ }^{, 10}$ En dicha definición, hace referencia al contexto temporal, de un presente con visión de futuro, en relación al quehacer del ser humano.

La Antropología Filosófica, estudia al ser humano como ser vivo capaz de reflexionar; capaz de "saber" (conocimiento). Sin embargo, el saber por sí sólo, no constituye absolutamente nada. El ser humano combina el saber con el hacer y el estar: saber-hacer, hacer-saber, saber-estar. Tal como lo describe Fernando Lolas en su libro de Bioética y Antropología Médica "Se sabe para hacer algo. Se hace algo para saber. Se hace lo que se sabe hacer." El saber estar "es la tonalidad moral del ejercicio de un saber y de la reflexión sobre el hacer. Quien sabe estar en lo suyo conoce sus límites, reconoce que lo factible no siempre es legítimo y que lo posible no siempre es necesario. Pone y se impone límites, reflexiona sobre normas, examina sus principios." ${ }^{, 11}$ Luego podemos concluir que gracias a la combinación de éstos el ser humano construye su vida y es capaz de comprenderse, al cuestionarse quién es, quién quiere ser y qué puede y/o tiene que realizar para logarlo; por 
medio de éstos comprende su historia y le da significado y sentido a su existencia. Reconoce la limitante del tiempo, por ende sabe que ha de planificar y diseñar su vida para poder llegar a ser, hacer y estar, de acuerdo a sus intereses y motivaciones. Este proceso de reflexión y análisis no es propio de un determinado momento en el ciclo vital del desarrollo humano, si no que es netamente personal. Así lo expresa José Luis Dell Ordine “el hombre, a partir de lo que es, se proyecta hacia lo que no es aún y desea ser. Estando determinado, viviendo en una situación concreta, en un aquí y un ahora, está impulsado a construirse así mismo, a ser él mismo con los otros...”. ${ }^{12}$

Tomando en consideración esta visión de la filosofía antropológica del ser humano, es que modificamos la definición de brecha ocupacional descrita por G. Eriksson. Brecha ocupacional es la variación que ocurre entre lo que un individuo quiere y necesita ser y/o hacer, y lo que él/ella realmente es y hace. La brecha/variación denota un cambio en el desempeño percibido por la persona de acuerdo a la variable temporal, es decir, de su desempeño actual, frente a una visión de su desempeño a futuro. Si bien se esclarece el delta en el desempeño ocupacional, ni la definición de brecha ocupacional, ni de N.O., da cuenta de las acciones o estrategias para alcanzar la situación deseada de la persona.

\section{Alterar:}

La Real Academia Española, establece alteración como “acción de cambiar la esencia o forma de algo."13

El hecho de no participar en ocupaciones significativas para la persona, genera una alteración en el sentimiento de satisfacción de vida, puesto que la persona se priva de aquellas ocupaciones que la definen y le otorgan identidad, que le producen placer y gratifican.

\section{Satisfacción de vida}

La satisfacción de vida es un concepto que suele confundirse con la calidad de vida y el bienestar. Si bien estos términos están interrelacionados, no poseen el mismo significado. La satisfacción con la vida es el componente cognitivo que engloba un juicio general (subjetivo y afectivo) de cómo la persona percibe su vida, independiente de las condiciones de vida objetiva ${ }^{14}$. Dicha percepción es generalmente evaluada por el nivel de bienestar y felicidad detectado por la persona en un momento y tiempo determinado.

Hay personas que están plenamente satisfechas con sus vidas, sin embargo éstas no les producen bienestar, en cuanto afectan directa o indirectamente su salud, y al ser evaluadas no poseen una buena calidad de vida.

\section{Modificar}

Según la Real Academia Española, se refiere a "limitar, determinar o restringir algo a cierto estado en que se singularice y distinga de otras cosas." ${ }^{15}$ La importancia de este término en la definición de N.O. se centra en la particularidad de que si bien hay un cambio en el desempeño de la persona y en las ocupaciones de su rutina diaria, éste puede no impedir su participación, mas no son valoradas de acuerdo al nivel de importancia, satisfacción y desempeño como quisiera la persona.

\section{Participación}

La CIF determina participación como "el acto de involucrarse en una situación vital. Representa la perspectiva de la sociedad respecto al funcionamiento. La participación 
denota el grado de implicación de la persona, lo cual incluye: tomar parte, ser incluido o participar en alguna área de la vida, así como las de ser aceptado, o tener acceso a los recursos necesarios, lo cual puede facilitar o obstaculizar la participación. En otras palabras la implicación hace referencia a la experiencia vivida por las personas en el contexto real en el que viven.

La presencia de una restricción en la participación viene determinada por la comparación de la participación de esa persona, con un estado de salud, con la participación esperable de las personas que no tuviesen un estado de salud similar al suyo (enfermedad, trastorno, daño, etc.).” 16

De acuerdo a esta definición, la participación no sólo toma en consideración que la persona desempeñe una determinada ocupación en un contexto temporo-espacialmente determinado, sino también considera como factores influyentes el acceso social y físico de su contexto de desempeño para poder participar, haciendo énfasis que la participación implica más que involucrarse en una actividad.

Al entender la participación como el acto de involucrarse en una situación vital; y las ocupaciones significativas, como aquellas actividades que son de gran valor para la persona, puesto que la definen e identifican, entonces podríamos decir que la restricción en la participación, corresponde a una limitación para desempeñar ocupaciones significativas.

\section{Ocupaciones significativas}

A lo largo de la evolución y desarrollo de la Terapia Ocupacional, se han creado numerosas definiciones del término “ocupación”. En ocasiones, al término “ocupación” se le adjudica un sustantivo calificativo para imprimirle un enfoque especial. Este es el caso de las ocupaciones significativas.

Mary Reilly, afirma que "existe una íntima relación entre el compromiso de las actividades significativas y la salud. Este tipo de ocupaciones están caracterizadas por el involucramiento e implicancia en actividades auto iniciadas, auto dirigidas que son vividas como productivas por la persona (mismo si el producto es la “diversión”) y contribuyen a otros." 17

"La ocupación significativa consiste en una oportunidad para el encuentro, el desencuentro y el reencuentro con uno mismo y con su entorno, a través de la apropiación del "hacer comprometido". Este método de trabajo beneficia la salud, en la medida que aproxima y concretiza la expresión de los intereses, valores y destrezas (habilidades y conocimientos) del sujeto."18

Si bien G. Eriksson no habla de ocupaciones significativas como tal, en la Tesis se describen las ocupaciones simbólicas como aquellas actividades que definen a la persona y que forman parte de su ser, siendo un medio para expresarse y reconocerse por medio del hacer.

Tras los textos citados podemos resumir que las ocupaciones significativas otorgan identidad a la persona, la definen y forman parte de su historia ocupacional, al ser auto iniciadas y auto dirigidas. Al desempeñarlas a través del "hacer comprometido" la persona promueve su salud y bienestar, expresando sus intereses, creencias y habilidades.

Tomando en cuenta estos conceptos y sus relaciones, podemos distinguir que las brechas ocupacionales generan NN.OO. en el individuo a través de un proceso de análisis, introspección, reflexión y/o toma de conciencia de su historia ocupacional. En donde la discrepancia o variación percibida producto del ser y/o hacer pasado v/s presente o presente 
v/s futuro, se traducen en una alteración del sentimiento de satisfacción de vida, que da cuenta de un cambio en el involucramiento de la persona en el desempeño de aquellas ocupaciones que eran consideradas y valoradas como significativas; comprometiendo su bienestar y su calidad de vida.

\section{METODO}

La investigación corresponde a un estudio de tipo exploratorio - descriptivo. Exploratorio, con el fin de recopilar información hasta la fecha no investigada, respecto a las NN.OO. de las madres de NANEAS en el contexto hospitalario y, en base a los datos recopilados determinar el impacto de éstas en el desempeño ocupacional de la madre, lo cual corresponde a la fase descriptiva de la investigación.

Objetivo general:

Determinar cómo impactan en el desempeño ocupacional, las necesidades ocupacionales de la madre cuidadora de un NANEAS durante el periodo de hospitalización.

Objetivos Específicos:

a. Identificar las necesidades ocupacionales percibidas por la madre cuidadora de un NANEAS durante el periodo de hospitalización.

b. Describir el impacto de las necesidades ocupacionales en el desempeño ocupacional de la madre cuidadora, durante el periodo de hospitalización.

Muestra:

La población a investigar corresponde a una madre cuidadora de su hija menor, quien, de acuerdo a los criterios, cumple con los requisitos de ser una NANEAS. La NANEAS fue hospitalizada en 4 oportunidades en el Hospital Clínico de la Universidad de Chile (y una vez en una Clínica), siendo la madre de ésta quien asume la responsabilidad de ser la cuidadora principal.

La obtención de resultados se llevó a cabo a través de la metodología de estudio de caso. Ésta fue escogida por la naturaleza del objeto de estudio (madre cuidadora principal de la NANEAS); por el nivel de control de las variables (no exige tener control sobre éstas, lo cual favorece nuestra investigación ya que las NN.OO. corresponden a un fenómeno subjetivo dado por la percepción de la persona) y la falta de investigaciones del tema. El recopilar información a través de esta metodología, permite considerar los múltiples aspectos que influyen en el sujeto de estudio, en profundidad.

Para la recopilación de datos, se aplicaron tres instrumentos: una entrevista semiestructurada, Patrón de Idiosincrasia (se aplica de acuerdo al contexto temporal: antes de la primera hospitalización, durante las hospitalizaciones y posterior al alta) y el Cuestionario de Necesidades Ocupacionales (se aplica con el mismo contexto temporal mencionado anteriormente). Para la aplicación de los distintos instrumentos, nos reunimos con la madre de la NANEAS, en cuatro instancias, éstas se llevaron a cabo durante el transcurso de un 
mes alternando los lugares de reunión entre el hogar de la madre de la NANEAS y la sala de espera del Policlínico de Pediatría del HCUCH.

En base a los instrumentos aplicados y la información obtenida, se llevó a cabo el análisis de los datos en base a las 5 áreas de la ocupación (Actividades de la vida diaria básicas e instrumentales, Trabajo, Educación, Participación social y Tiempo Libre.), la percepción de N.O. y las modificaciones realizadas para el desempeño de la ocupación.

\section{RECOLECCIÓN Y ANÁLISIS DE DATOS}

\section{Acercamiento al caso}

La madre en estudio es una mujer de 44 años, casada hace 19 años con un hombre de 52 años (profesional), con quien tiene dos hijas (16 y 15 años). La familia posee un estrato socio-económico medio y pertenece al sistema de salud privado. La madre trabaja en empleo informal independiente no remunerado (cumple funciones de secretaria para empleo independiente de su esposo, además de participar en actividades voluntarias, tales como el equipo de solidaridad del colegio de sus hijas). La familia vive en una casa de dos pisos en la ciudad satelital de Maipú. Las hijas asisten a un colegio privado ubicado en las cercanías del hogar.

La madre posee una red social operante pequeña (compuesta principalmente por su familia nuclear: 3 personas, dos amigas que son sus vecinas y el apoyo espiritual brindado por la comunidad religiosa), pero eficiente.

En Julio del año 2007 la hija menor de la familia comienza con algunos síntomas de Miastemia Gravis, los cuales son atribuidos a un cuadro de estrés. En Noviembre del mismo año, durante una intervención de ortodoncia, la menor sufre un shock anafiláctico, agonizando durante 5 horas, dando inicio a un proceso de hospitalizaciones reiteradas en donde la condición de salud de la menor era altamente vulnerable y propicia al contagio de infecciones. En resumen fueron 5 hospitalizaciones en un periodo de 5 meses. Durante los periodos de hospitalización de la NANEAS, la madre asume el rol de cuidadora principal de ésta, modificando y postergando su participación en las ocupaciones de su rutina diaria.

\section{Análisis de Datos Fase Exploratoria: conociendo modificaciones en la rutina.}

Tras la recopilación de los datos pudimos constatar las modificaciones en las ocupaciones de la vida diaria que realiza la madre para responder a las demandas del medio. Para el análisis correspondiente se eligieron 3 ejes de estudio: contexto, roles y desempeño ocupacional. Los ejes mencionados nos permiten comprender y establecer relaciones causales entre las variables en estudio. En base a lo anterior se observa lo siguiente:

\section{Contexto}

Los contextos de desempeño en los cuales la madre generalmente se desenvolvía eran: personal, sociocultural, físico, espiritual y virtual. Ahora bien, antes de la primera hospitalización los contextos que la madre identifica como más significativos son: el físico (el hogar), el sociocultural (participación en actividades relacionadas con el colegio de las 
hijas y red de apoyo: amigas) y el espiritual (asistir a la parroquia). Durante las hospitalizaciones de la NANEAS, el principal contexto de desempeño a nivel físico es el hospital. Su participación en los contextos espiritual (cadenas de oración) y sociocultural (con la red de apoyo operante mediante el uso de dispositivos de comunicación y estableciendo vínculos con el personal del hospital), es modificada. El contexto virtual adquiere mayor relevancia, con el uso de dispositivos de la comunicación y con la exploración de actividades de tiempo libre, como es el caso de ver televisión.

\section{Roles}

Aún cuando los roles ocupacionales que ejercía el sujeto en estudio previo a los periodos de hospitalización eran el rol de: madre, esposa, dueña de casa, amiga y rol laboral; no todos poseían el mismo valor. Es más, algunos roles, como el caso del rol de madre y dueña de casa estructuraban y organizaban su rutina diaria. Al momento de adquirir el rol de cuidadora principal, la madre modifica el desempeño de su rutina de acuerdo a las demandas que le genera dicho rol en el contexto hospitalario, en donde permanece las 24 horas del día. El hecho de asumir el nuevo rol, y desenvolverse en el contexto hospitalario conducen a la madre a postergar las funciones que le demanda su rol laboral; no así en el caso de su rol de madre, esposa, dueña de casa y amiga, puesto que las tareas de éstos son modificados (acude a su red social operante para desempeñar las funciones que ella no puede llevar a cabo presencialmente, y en el caso de su rol de amiga, éste se limita al contacto telefónico).

\section{Área del desempeño de la ocupación}

Antes de la primera hospitalización el sujeto en estudio manifiesta a través de los instrumentos aplicados poseer un equilibrio ocupacional en relación a las ocupaciones que conforman su vida diaria y a las áreas de desempeño de éstas, aún cuando las AVD instrumentales asociadas a su rol de madre y dueña de casa, estructuraban su rutina diaria. Cuando comienzan las hospitalizaciones de la NANEAS, se observa un quiebre en este equilibrio, y no sólo por la deprivación ocupacional que significa el contexto hospitalario, sino también por el hecho que la madre al asumir el rol de cuidadora principal permanece con la NANEAS las 24 horas del día. En este contexto y en relación a las áreas del desempeño que componen su rutina observamos lo siguiente (ver Tabla 1).

De las AVD básicas, el cuidado de dispositivos personales (para lo cual disponía de un casillero con candado) y el dormir/descansar (N.O. que surge previo a la hospitalización y que es cubierta en el hospital), adquieren un nivel mayor de importancia. La N.O. del vestuario conlleva a modificar su desempeño con el objetivo de satisfacerla (coordina telefónicamente con sus amigas el lavado y planchado de su ropa diariamente, para que su esposo se la entregue en las visitas diarias). La actividades de alimentación/comer y la actividad sexual, son postergadas por el hecho de permanecer junto a la NANEAS todo el día.

La mayor parte de las AVD instrumentales son delegadas a la red de apoyo operante por medio del uso de dispositivos de la comunicación (ocupación que adquiere especial importancia en este contexto), actividades tales como: vestuario, organización y manejo del 
hogar, cuidado de otros (en este caso de la hija mayor), cuidados de la mascota, compras y preparación de comidas. Se observa claramente cómo adquieren significado e importancia las ocupaciones de: procedimientos de seguridad y respuesta a emergencia (contratan un servicio de ambulancia para los casos de emergencia), el cuidado de otros (de la NANEAS) y el mencionado uso de dispositivos de comunicación (uso del teléfono celular). Las otras ocupaciones (manejo de finanzas, crianza de los hijos, traslado en la comunidad), las sigue desempeñando pero el modo de ejecutarlas no es el mismo que antes. En el caso del manejo y mantención de su salud, es postergado (deja de asistir a sus controles médicos).

Las ocupaciones significativas relacionadas al área de Trabajo y Tiempo Libre que desempeñaba previo a las hospitalizaciones, son postergadas. Ahora bien, respecto al Tiempo Libre, explora su participación en una nueva actividad: ver televisión. Actividad que con el tiempo comienza a ser significativa para la madre.

Su desempeño en el área de Participación Social es modificada, limitándose a: las visitas diarias de su esposo (la visita media hora en la mañana y una hora en la tarde), el contacto telefónico con su red de apoyo y a establecer vínculos con el personal del hospital (cuidadores, personal de enfermería, de aseo, profesionales, entre otros).

En el área Educación, la madre percibe la necesidad de aprender sobre cuidados básicos del enfermo y primeros auxilios (por la condición de salud vulnerable de la NANEAS), además de obtener la licencia de conducir.

Tabla 1: Necesidades Ocupacionales del contexto hospitalario.

\begin{tabular}{|c|c|}
\hline Áreas del desempeño & Ocupaciones percibidas como N.O. en el contexto hospitalario \\
\hline AVD básica & $\begin{array}{c}\text { Vestuario } \rightarrow \text { cubierta } \\
\text { Alimentación/comer } \rightarrow \text { no cubierta }\end{array}$ \\
\hline AVD instrumental & $\begin{array}{c}\text { Manejo de finanzas } \rightarrow \text { cubierta } \\
\text { Crianza de las hijas } \rightarrow \text { no cubierta } \\
\text { Manejo y manutención de la salud } \rightarrow \text { no cubierta } \\
\text { Cuidado de otros } \rightarrow \text { cubierta } \\
\text { Procedimiento de seguridad y respuesta a emergencias } \rightarrow \text { cubierta } \\
\text { Uso de dispositivos de la comunicación } \rightarrow \text { cubierta }\end{array}$ \\
\hline Educación & $\begin{array}{c}\text { Aprender sobre cuidados básicos del enfermo y primeros auxilios } \rightarrow \text { no cubierta } \\
\text { Aprender a manejar para obtener la licencia de conducir } \rightarrow \text { no cubierta }\end{array}$ \\
\hline
\end{tabular}

\section{Análisis de Datos Fase Descriptiva: identificando necesidades ocupacionales.}

Los resultados obtenidos en la fase descriptiva se expondrán en torno a la identificación de NN.OO. percibidas por la madre a través de su narrativa, los Patrones de Idiosincrasia y el Cuestionario de Necesidades Ocupacionales; y en base a éstos las modificaciones o adaptaciones realizadas en las ocupaciones de la vida diaria que componen su rutina.

En el caso estudiado, podemos ver cómo los roles y contextos en que se desenvuelve la madre se interrelacionan, dando origen a las NN.OO. Es así como las ocupaciones de la vida diaria que conforman la rutina de la madre, van definiendo las funciones de sus roles ocupacionales las cuales se ven modificadas al momento de asumir el rol de cuidadora principal de la NANEAS en el contexto hospitalario. 
Previo a las hospitalizaciones de la NANEAS, los roles más significativos para la madre y que estructuraban y organizaban su rutina, eran el rol de madre y dueña de casa. Durante los periodos de hospitalización, a éstos se les agrega el rol de cuidadora principal de la NANEAS. En este contexto, para la madre se hace vital la necesidad de modificar su participación en la ejecución de las demandas de cada una de las funciones asociadas a sus roles ocupacionales, con el fin de cumplir con los requerimientos de ser la cuidadora principal de su hija y permanecer a su lado las 24 horas del día.

Luego de conocer las variaciones en las ocupaciones de la vida diaria de la madre, es indispensable conocer cómo impactan las NN.OO. surgidas en el contexto hospitalario y que no son cubiertas por la madre. Las ocupaciones a analizar entonces, se restringen a:

> AVD básicas: vestuario, alimentación/comer.

$>$ AVD instrumentales: crianza de la hija mayor, manejo y mantención de su salud, cuidado de otros (de la NANEAS), procedimiento de seguridad y respuesta a emergencias, manejo de finanzas y uso de dispositivos de comunicación.

$>$ Educación: aprender sobre cuidados básicos de un enfermo y primeros auxilios, y obtener licencia de conducir.

De las NN.OO. surgidas y percibidas en el contexto hospitalario, no logra satisfacer de las AVD básicas: la alimentación/comer; de las AVD instrumentales: la crianza de la hija mayor y manejo y mantención de su salud; y las relacionadas al área de educación.

En relación a las actividades de alimentación/comer y educación, se conforman como NN.OO., gracias a la influencia que el contexto socio-cultural del hospital ejerce sobre la madre. Durante los periodos de hospitalización las enfermeras y auxiliares del hospital (agentes externos) logran que la madre modifique su participación en la ocupación de alimentación/comer, visualizándola como una actividad imprescindible para mantener su condición de salud. Además le brindan la oportunidad de participar de manera exploratoria en una instancia educativa informal, a través de la observación o imitación de los cuidados que le entregan a la menor. Esta instancia genera la N.O. de formarse en cuidados básicos del enfermo y primeros auxilios, con el objetivo de satisfacer las demandas de su rol de cuidadora principal de la NANEAS.

Considerando todos los esfuerzos de la madre por cubrir la N.O. en relación a la crianza de su hija mayor, las acciones realizadas no cubren en su totalidad la brecha percibida, pues identifica que su participación en esta ocupación no es totalmente satisfactoria ni su desempeño el mejor, refiriendo sentimientos de culpa al no haber estado presente en instancias importantes de la vida de su hija mayor, ocurridas durante los periodos de hospitalización.

Si bien la N.O. de manejo y mantención de su salud altera el sentimiento de satisfacción de vida, ésta no genera un cambio en el comportamiento ocupacional, puesto que la madre adopta una actitud pasiva.

Finalmente las actividades de vestuario, cuidado de otros (de la NANEAS) y uso de dispositivos de la comunicación, manejo de finanzas y procedimientos de seguridad y respuesta a emergencias logran ser cubiertas durante el periodo hospitalario. 


\section{RESULTADOS}

Luego de terminar el proceso de análisis de datos podemos ver que la madre en estudio durante los periodos de hospitalización logró cubrir gran parte de sus NN.OO., tomando una actitud activa en la búsqueda de estrategias que le permitieran satisfacer las NN.OO. percibidas; favoreciendo el desempeño de sus roles ocupacionales significativos.

El hecho de asumir el rol de cuidadora principal de la NANEAS y permanecer las 24 hrs. junto a ella, provoca que el contexto hospitalario sea el principal medio donde se desenvuelve la madre. En consecuencia de lo anterior, la participación en sus ocupaciones de la vida diaria y en las funciones de sus roles son modificadas. Aún cuando en los periodos de alta hospitalaria, la madre vuelve a su hogar y a retomar su rutina, ésta no era igual a la que tenía previo a los periodos de hospitalización, ya que la NANEAS requería cuidados especiales, por ende, la madre continúa modificando su participación, con el objetivo de satisfacer las necesidades de su rol de cuidadora. En resumen, podemos constatar que los roles ocupacionales definen las ocupaciones de la vida diaria, siendo ésta última influenciada por el contexto en donde se desempeña la rutina. Luego, las NN.OO. del caso en estudio surgen del cambio en el contexto y en la rutina de la madre, dado por los cambios en el rol ocupacional. Además de las variables del entorno, también es importante considerar las variables propias del sujeto de investigación, pues éstas influyen en la percepción y detección de las N.O.; y en consecuencia a esto, en la actitud que la persona adopta en relación a éstas. En este caso, la madre de la NANEAS se adapta sin problemas a las condiciones del contexto hospitalario; e incluso crea estrategias para cubrir las N.O. percibidas, (las cuales estaban íntimamente relacionadas con el rol de cuidadora principal y las ocupaciones de la vida diaria, relacionadas principalmente con las AVD instrumentales). Entonces, podemos afirmar que las ocupaciones relacionadas con el desempeño del rol de madre, dueña de casa y cuidadora, corresponden a ocupaciones significativas, que le otorgan un importante sentimiento de satisfacción de vida, y por lo mismo, corresponden a una prioridad de participación en su quehacer ocupacional a lo largo de su historia. Algunos de los factores protectores que facilitaron a la madre la ejecución de su rol de cuidadora principal de la NANEAS fueron: poseer una red social de apoyo operante activa, su situación económica (permite acceder a servicio de ambulancia privada, aumentar plan de teléfono celular y finalmente le da la posibilidad de abandonar su trabajo para dedicarse al cuidado de su hija) y el tipo de trabajo que desempeñaba (trabajo de tipo informal).

La madre modificó su participación en la mayoría de las ocupaciones de su vida diaria, que le eran significativas sin percibir grandes brechas ocupacionales y por ende, sin generar alteraciones importantes en su sentimiento de satisfacción de vida. Aquellas ocupaciones significativas que deja de desempeñar, las detecta como NN.OO. que pretende satisfacer a futuro. Si bien percibe la importancia personal que tienen en su rutina diaria y en su historia de vida dichas ocupaciones, y aún cuando tiene el deseo de volver a desempeñarlas, la alteración en el sentimiento de satisfacción de vida no es lo suficientemente importante como para movilizarla a la acción, es decir, a adoptar una postura activa que genere un cambio en su comportamiento ocupacional.

Las áreas ocupacionales en las que se produce mayor impacto son en las AVD instrumentales, Trabajo y Tiempo Libre. En cada una de estas áreas la madre refleja un 
cambio en el nivel de satisfacción, importancia y desempeño (ver Tabla 2), alterando de distintas maneras su participación y su sentimiento de satisfacción de vida. En el caso de las AVD instrumentales la madre identifica cambios en el desempeño de las actividades antes y durante la hospitalización de su hija. A diferencia de éstas, en las áreas de Trabajo y Tiempo Libre son postergadas durante los periodos de hospitalización. Esta decisión está marcada por las condiciones que demanda el desempeño de su rol de cuidadora principal de la NANEAS en el contexto hospitalario. La N.O. percibida en este caso, no produce en la madre la motivación y energía necesaria para generar modificaciones que le permitan continuar con su desempeño, pues existen otras ocupaciones que tienen mayor significado. No así con la Participación Social, que le demanda a la madre crear estrategias para cubrirlas.

Ahora bien, el hecho de cubrir una N.O. no siempre posee efectos positivos sobre el desempeño ocupacional, puesto que los medios utilizados para satisfacerlas, no siempre promueven el bienestar y calidad de vida. En el caso de la madre en estudio, la necesidad de cuidar a la NANEAS llega a tal punto, que la madre deja de alimentarse y de asistir a sus chequeos médicos, por permanecer junto a ella durante todo el día.

Con respecto a la metodología de aplicación de los instrumentos, en los resultados se reflejaron contradicciones en la valoración de las ocupaciones de la vida diaria. Motivo por el cual, se ve la necesidad de sistematizar la aplicación del concepto y sus instrumentos (Patrones de Idiosincrasia, entrevista semi-estructurada y Cuestionario de Necesidades Ocupacionales), con el fin de evitar la aparición de estos sesgos que esconden los resultados reales y dificultan el proceso de análisis de datos.

En el transcurso de la aplicación de los instrumentos la madre reflexionó en torno a su rutina pasada (antes y durante las hospitalizaciones) y su rutina actual, contrastando estas dos realidades. Lo anterior con el objetivo de hacerla percibir e identificar las NN.OO. que surgieron en el contexto hospitalario. Finalmente, el proceso de recolección de datos, se convirtió en una instancia de intervención, la cual permitió el reconocimiento de las NN.OO. La madre es capaz de identificar ocupaciones significativas que fueron postergadas, siendo consideradas para ser retomadas a la brevedad posible, por el nivel de satisfacción que le proporciona su participación y desempeño. Dicho proceso fue avalado por el médico tratante de la NANEAS, visualizando la participación de la madre en el estudio como una instancia terapéutica beneficiosa que le permitió exteriorizar la vivencia. 
Tabla 2: Extracto de la Aplicación del Cuestionario de Necesidades Ocupacionales

\begin{tabular}{|c|c|c|c|c|c|c|c|c|c|c|}
\hline \multicolumn{11}{|c|}{ CUESTIONARIO SOBRE NECESIDADES OCUPACIONALES } \\
\hline \multirow{4}{*}{ OCUPACIÓN } & \multicolumn{4}{|c|}{ LO HACÍA (antes de la hospitalización) } & \multicolumn{4}{|c|}{ LO HAGO (durante la hospitalización) } & \multirow{3}{*}{\multicolumn{2}{|c|}{\begin{tabular}{l}
\multicolumn{1}{c}{ ME } \\
GUSTARÍA \\
HACERLO
\end{tabular}}} \\
\hline & \multirow{3}{*}{ Importancia } & \multicolumn{2}{|c|}{$\mathrm{Si}$} & \multirow{3}{*}{ No } & \multicolumn{3}{|c|}{ Si } & \multirow[t]{3}{*}{ No } & & \\
\hline & & & & & \multirow{2}{*}{ Importancia } & \multirow{2}{*}{ Satisfacción } & \multirow{2}{*}{ Desempeño } & & & \\
\hline & & & & & & & & & $\mathbf{S i}$ & No \\
\hline \multicolumn{11}{|l|}{ AVD BÁSICAS } \\
\hline Vestuario & 10 & 10 & 10 & & 10 & 10 & 10 & & $\mathrm{X}$ & \\
\hline Comer/Alimentarse & 5 & 5 & 5 & & 5 & 5 & 5 & & $\mathrm{X}$ & \\
\hline Actividad sexual & 10 & 10 & 10 & & & & & $\mathrm{X}$ & $\mathrm{X}$ & \\
\hline Dormir/Descansar & 10 & 10 & 4 & & 10 & 10 & 10 & & $\mathrm{x}$ & \\
\hline \multicolumn{11}{|l|}{$\begin{array}{c}\text { AVD } \\
\text { INSTRUMENTALES }\end{array}$} \\
\hline Ir de compras & 5 & 5 & 10 & & 1 & 3 & 1 & & & $\mathrm{X}$ \\
\hline $\begin{array}{l}\text { Administración } \\
\text { finanzas }\end{array}$ & 10 & 10 & 10 & & 10 & 10 & 10 & & $\mathrm{X}$ & \\
\hline $\begin{array}{c}\text { Procedimientos y } \\
\text { respuesta a } \\
\text { emergencias }\end{array}$ & 10 & 10 & 10 & & 10 & 10 & 10 & & $\mathrm{X}$ & \\
\hline $\begin{array}{c}\text { Preparación de } \\
\text { comidas y limpieza }\end{array}$ & 5 & 3 & 5 & & 1 & 1 & 1 & & $\mathrm{X}$ & \\
\hline $\begin{array}{c}\text { Organización y manejo } \\
\text { del hogar }\end{array}$ & 10 & 10 & 10 & & 10 & 10 & 10 & & $\mathrm{X}$ & \\
\hline $\begin{array}{c}\text { Manejo y manutención } \\
\text { de salud }\end{array}$ & 10 & 10 & 10 & & 10 & 10 & 10 & & $\mathrm{X}$ & \\
\hline Uso transporte público & 8 & 8 & 10 & & 10 & 3 & 10 & & $\mathrm{X}$ & \\
\hline Uso transporte privado & 3 & 5 & 1 & & 10 & 10 & 1 & & $\mathrm{X}$ & \\
\hline Crianza de los hijas & 10 & 10 & 10 & & 10 & 10 & 8 & & $\mathrm{X}$ & \\
\hline Cuidado de otros & 10 & 10 & 10 & & 10 & 10 & 8 & & $\mathrm{X}$ & \\
\hline Cuidado de la mascota & 10 & 10 & 10 & & 10 & 10 & 8 & & $\mathrm{X}$ & \\
\hline \multicolumn{11}{|l|}{$\begin{array}{l}\text { PARTICIPACIÓN } \\
\text { SOCIAL }\end{array}$} \\
\hline $\begin{array}{c}\text { Estar con la pareja y } \\
\text { los hijos }\end{array}$ & 10 & 10 & 10 & & 10 & 8 & 9 & & $\mathrm{X}$ & \\
\hline Actividades religiosas & 10 & 10 & 10 & & 10 & 10 & 10 & & $\mathrm{X}$ & \\
\hline Viajar por placer & 10 & 10 & 10 & & 1 & 1 & 1 & $X$ & $\mathrm{X}$ & \\
\hline Actividades escolares & 10 & 10 & 10 & & 1 & 1 & 1 & $\mathrm{X}$ & $\mathrm{x}$ & \\
\hline $\begin{array}{c}\text { TRABAJO } \\
\text { Trabajo sin } \\
\text { remuneración / } \\
\text { voluntariado } \\
\end{array}$ & 10 & 10 & 10 & & 1 & 1 & 1 & $\mathrm{X}$ & $\mathrm{X}$ & \\
\hline
\end{tabular}

\section{DISCUSIÓN}

Los modelos teóricos utilizados y la actual mirada de la Terapia Ocupacional como disciplina, ven a la persona como un sujeto indivisible del ambiente en donde ocurre constantemente una ocupación, es decir, de la interacción de la persona con el ambiente sucede la ocupación. Dicho postulado es lo que se demuestra en la definición del concepto de Necesidad Ocupacional; puesto que la percepción de la brecha y el sentimiento de satisfacción de vida de la persona, modifican su participación en el contexto en relación al 
desempeño de ocupaciones significativas, entregando una clara panorámica de cómo estos tres elementos se conjugan en un momento y espacio determinado.

Tras un proceso de reflexión e introspección, el T.O. puede guiar o facilitar a la persona a cubrir la N.O. detectada desde una perspectiva del quehacer profesional, a modo que la satisfacción de la N.O., favorezca la salud, el bienestar y la calidad de vida de la persona, enriqueciendo su desempeño ocupacional al involucrarla en ocupaciones significativas. En el caso de la madre, algunas de las NN.OO. surgieron luego de un proceso influenciado por un agente externo que la hace reflexionar. Como es el caso de los hábitos saludables de alimentación, manejo y manutención de su salud y de aprender sobre cuidados básicos del enfermo y primeros auxilios. Gracias a esto, la madre es capaz de percibir la N.O., en donde las brechas percibidas, la movilizan a modificar su participación en dichas áreas.

Una persona ante una N.O. puede adoptar dos posturas: pasiva o activa. La pasiva, se produce cuando la persona o el medio no le permite a ésta cubrir la N.O. percibida, ya sea por factores personales (como por ejemplo: falta de habilidades y destrezas, alteración en las estructuras y funcionamiento, alteraciones en el autoconcepto, o bien la alteración en el sentimiento de satisfacción de vida no genera el impacto suficiente para movilizar a la persona al cambio); o factores contextuales (como por ejemplo: falta de recursos, y/o acceso a los medios requeridos para satisfacer las necesidades, entre otras). En cuanto a la activa, corresponde a la movilización de la persona a satisfacer la N.O. percibida, usando recursos personales o del entorno. Lo anterior corresponde al concepto de causalidad personal descrito por Kielhöfner en el Modelo de Ocupación Humana ${ }^{7}$. Dichas posturas no son mutuamente excluyentes en el tiempo, es decir, el individuo puede oscilar entre una y otra dependiendo de factores propios del sujeto como del contexto que lo rodea, en un periodo determinado.

La aplicación del concepto de Necesidad Ocupacional, se convirtió en una herramienta de diagnóstico e intervención en el caso en estudio, en donde la madre logra identificar los cambios realizados en las ocupaciones que conforman su rutina diaria, visualizando las modificaciones de sus roles ocupacionales, al desenvolverse en otro contexto de desempeño. 


\section{REFERENCIAS BIBLIOGRAFICAS}

${ }^{1}$ MCPHERSON M., ARANGO P., FOX H., et al. A New Definition of Children with Special Health Care Needs. Pediatrics. 1998; 102: 137-140.

${ }^{2}$ SIMÓ, S.; URBANOWSKI, R.; El Modelo Canadiense del Desempeño Ocupacional I. Revista Gallega de Terapia Ocupacional. Febrero 2006. Vol. 3, 1-27.

${ }^{3}$ DE LAS HERAS C., Teoría y Aplicación del Modelo de Ocupación Humana, Primera parte. Reencuentros. 2004.

4 AOTA, Occupational Therapy practice Framework: Domain And Process. Revista Americana de Terapia Ocupacional, Noviembre/diciembre 2002. 56, 609-639.

${ }^{5}$ KIELHOFNER G., Conceptual Foundations of Occupational Therapy. F.A. Davis Company. Philadelphia. 1992; 10: 154 - 169.

${ }^{6}$ HEARD, CHYNTHIA; Occupational role acquisition: A perspective on the chronically disabled. AJOT. Abril 1977. 31, 243 - 247.

${ }^{7}$ BURKE, J.P..; A clinical perspective on motivation: Pawn versus origin. AJOT. Abril 1977. 31, 254-258.

${ }^{8}$ ECHEVERRÍA A., ORTEGA P., Necesidades Ocupacionales en las Madres Cuidadoras de un Niño/a y/o Adolescente Con Necesidades Especiales de Atención en Salud, durante su Hospitalización. [Seminario de Título], Santiago, Chile, Universidad de Chile. 2008.

${ }^{9}$ CAPPONI R. Psicología, Psicopatología y Semiología Psiquiátrica en: Psicopatología y Semiología Psiquiátrica. $2^{\circ}$ ed. Santiago, Chile. Editorial Universitaria; 1992.

${ }^{10}$ ERIKSSON, G., Occupational Gaps after Acquired Brain Injury: An Exploration Of Participation In Everyday Occupations And The Relation To Life Satisfaction. Ed. Karolinska Institute. 2007.

11 LOLAS STEPKE, F., Saber, Saber-Hacer y Saber-Estar Hacia una ética del conocimiento. Bioética y Antropología Médica. Editorial Mediterráneo. 2000; 23-24.

${ }^{12}$ DELL ORDINE, J.L.; Introducción a la Antropología Filosófica. Portal Alipso [serie on line] Octubre 2002: [5 pantallas]. Disponible en: URL:

http://www.adipso.com/monografias/antropologia_filosofica.

${ }^{13}$ Diccionario Real Academia Española. España: 22 Edición; 2001. Alterar. 
${ }^{14}$ BEREZIN, S.; CHARDON, C.; DEMIRYI, M.; SUTER, M Y PELLEGRINI, M., et al. Promoción De La Salud En Espacios Educativos - Universitarios, ¿Utopía O Nuevos Desafíos? Universidad Nacional de Quilmas Argentina. Revista Terapia Ocupacional Galicia [serie on line] Septiembre 2005; (2): [6 pantallas]. Disponible en: URL: http://www.revistatog.com/num2/num2art1.htm.

${ }^{15}$ Diccionario Real Academia Española. España: 22 Edición; 2001. Modificar.

${ }^{16}$ OMS. CIF, 2001.

${ }^{17}$ BEREZIN, S.; CHARDON, C.; DEMIRYI, M.; SUTER, M Y PELLEGRINI, M., et al. Promoción De La Salud En Espacios Educativos - Universitarios, ¿Utopía O Nuevos Desafíos? Universidad Nacional de Quilmas. Argentina. Revista Terapia Ocupacional Galicia [serie on line] Septiembre 2005; (2): [6 pantallas]. Disponible en: URL: http://www.revistatog.com/num2/num2art1.htm.

${ }^{18}$ VIANA MOLDES, I.; GARCÍA PINTO, M.C.; ÁVILA ÁLVAREZ, A.; Ocupación Alienante versus Ocupación Significativa. Artículo Portal español de Terapia Ocupacional [serie on line]; [10 pantallas]. Disponible en: URL: http://www.terapiaocupacional.com/articulos/Ocup_Alienan_vs_ocup_signi.shtml. 